\title{
Hélène Lazure
}

Professeure agrégée, Faculté des sciences infirmières, Université de Montréal

(1985)

\section{"L’infirmière"}

Un document produit en version numérique par Jean-Marie Tremblay, bénévole, Professeur sociologie au Cégep de Chicoutimi

Courriel: jean-marie_tremblay@uqac.ca

Dans le cadre de "Les classiques des sciences sociales" Site web: http://classiques.uqac.ca/

Une bibliothèque fondée et dirigée par Jean-Marie Tremblay, sociologue

Une collection développée en collaboration avec la Bibliothèque

Paul-Émile-Boulet de l'Université du Québec à Chicoutimi

Site web: http://bibliotheque.uqac.ca/ 
Cette édition électronique a été réalisée Jean-Marie Tremblay, bénévole, professeur de soins infirmiers retraitée de l'enseignement au Cégep de Chicoutimi

Courriel: jean-marie_tremblay@uqac.ca

à partir du texte de :

Hélène Lazure, “L’infirmière”. Un article publié dans l'ouvrage sous la direction de Jacques Dufresne, Fernand Dumont et Yves Martin, Traité d'anthropologie médicale. L'Institution de la santé et de la maladie. Chapitre 30, pp. 631-643. Québec: Les Presses de l'Université du Québec, l'Institut québécois de recherche sur la culture (IQRC), Presses de l'Université de Lyon, 1985, 1245 pp.

Mme Lazure est professeure agrégée, Faculté des sciences infirmières, Université de Montréal

[Autorisation formelle de l'auteure de diffuser cet article dans Les Classiques des sciences sociales accordée le 27 mai 2006.]

13

Courriel : helene.lazure@umontreal.ca

Polices de caractères utilisée :

Pour le texte: Times New Roman, 14 points.

Pour les citations : Times New Roman 12 points.

Pour les notes de bas de page : Times New Roman, 12 points.

Édition électronique réalisée avec le traitement de textes Microsoft Word 2004 pour Macintosh.

Mise en page sur papier format : LETTRE (US letter), 8.5’’ x 11'’)

Édition numérique réalisée le 27 mai 2006 à Chicoutimi, Ville de Saguenay, province de Québec, Canada.

\section{Fait avec \\ Macintosh}




\section{Hélène Lazure}

Professeure agrégée, Faculté des sciences infirmières,

Université de Montréal
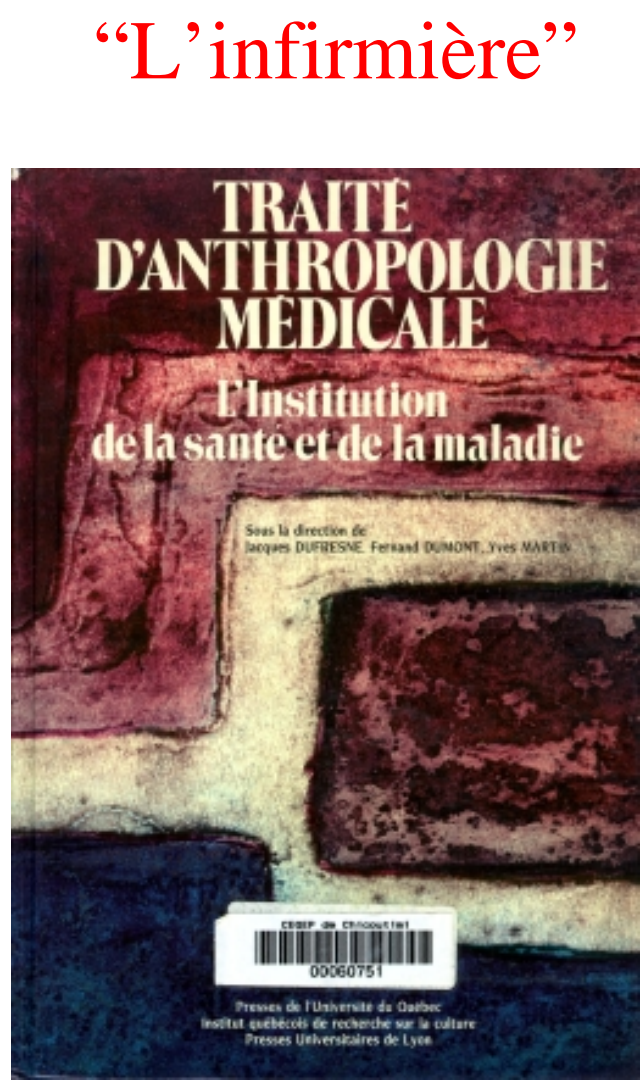

Un article publié dans l'ouvrage sous la direction de Jacques Dufresne, Fernand Dumont et Yves Martin, Traité d'anthropologie médicale. L'Institution de la santé et de la maladie. Chapitre 30, pp. 631-643. Québec: Les Presses de l'Université du Québec, l'Institut québécois de recherche sur la culture (IQRC), Presses de l'Université de Lyon, 1985, 1245 pp. 


\section{Table des matières}

$\underline{\text { Introduction }}$

Le soin : survivance

Naissance de la première infirmière

$\underline{\text { L'infirmière et le médecin }}$

L'influence du christianisme

De la lampe au phare: Florence Nightingale

Soigner : ancrer à la vie

Des soins avec ou sans bon sens

Une expérience à tenter

L'infirmière en devenir

Non à la profession omnibus

Oui à la vie

$\underline{\text { Références }}$ 
Hélène Lazure, “L’infirmière”.

Un article publié dans l'ouvrage sous la direction de Jacques Dufresne, Fernand Dumont et Yves Martin, Traité d'anthropologie médicale. L'Institution de la santé et de la maladie. Chapitre 30, pp. 631-643. Québec: Les Presses de l'Université du Québec, l'Institut québécois de recherche sur la culture (IQRC), Presses de l'Université de Lyon, 1985, 1245 pp.

\section{Introduction}

$\underline{\text { Retour à la table des matières }}$

Comment résister à la tentation de faire un lien entre l'infirmière, les soins infirmiers et l'expression bien connue : "Vivre d'amour et d'eau fraîche ». Indépendamment du sens précis qu'on lui attribue généralement, cette expression situe l'amour à l'instar de l'eau, élément physique vital, dans le rôle essentiel de nourrice de la vie. Il en est de même pour le genre humain qui sans l'apport de soins inspirés par l'amour, n'aurait pu développer et maintenir les interactions essentielles à son solide enracinement dans la vie.

\section{Le soin : survivance}

L'homme des cavernes est né, il s'est battu pour se nourrir et conquérir son environnement; en cela, il nous a tracé la voie. L'homme des cavernes a survécu et s'est perpétué ; il a évolué au prix de blessures, de souffrances et même de sa propre mort. L'homme des cavernes n'était pas seul dans son aventure. Au tout début, son instinct de survie lui dictait l'art de soigner sa progéniture, puis son entourage pansa ses plaies, se substitua à lui dans la quête de nourriture et de 
chaleur en attendant qu'il puisse à nouveau se battre pour assurer vaillamment la subsistance de chacun et prodiguer des soins à son tour. La survivance même de l'espèce humaine dépendait déjà des soins qu'elle était en mesure de se donner.

À cette époque, le rôle de soignant ne devait être qu'une facette de la polyvalence nécessaire à l'homme et les habiletés reliées à ce rôle devaient se calquer sur l'empirisme utilisé dans la conquête vitale de l'environnement. Bien avant d'être érigé en métier, le soin de l'homme a constitué un aspect capital de son évolution.

Tout ce qui précède n'est qu'hypothèse, dira-t-on. Le vide créé par le silence sur cette époque archaïque attise l'imagination des uns et le sentiment de frustration des autres. Notre unique point de repère, bien vérifiable celui-là, c'est que nous sommes tous là actuellement. Mais à quel prix ? Notre vulnérabilité face à la nature s'est vue compensée par notre exceptionnelle capacité d'adaptation greffée à la charpente des soins essentiels à la continuité de la vie et ce, en dépit des multiples agressions qui fusent à l'aube d'une civilisation.

\section{Naissance de la première infirmière}

\section{$\underline{\text { Retour à la table des matières }}$}

Heureux d'avoir échappé à la menace d'avortement de l'espèce, l'homme crut devoir raffiner son mode de vie dans le but d'accroître son efficacité. Les femmes au centre, les hommes dehors : c'est la consigne que l'on donne dans maintes danses folkloriques québécoises, mais c'était aussi un des premiers principes de l'organisation sociale. L'homme dirigeait son activité vers la maîtrise de la nature environnante et la femme y participait, n'en déplaise aux féministes, en donnant la vie et en créant les conditions nécessaires à son maintien et à la restauration des forces et de la santé. Ainsi le rôle et les habiletés de soignant commencent à se différencier par une transposition élargie du rôle maternel dont le lieu privilégié d'exercice fut d'abord la famille pour s'étendre ensuite à la communauté. 
$\mathrm{Si}$, jusqu'à tout récemment, il était commode de croire que la compétence maternelle était instinctive, on eut tôt fait de constater qu'être mère n'offrait pas une garantie à toute épreuve pour entrer en contact avec les individus et les aider à traverser, sinon transcender, les crises inhérentes à toute vie humaine. Le rôle de soignant allait désormais être assumé par celles qui en avaient le désir et les habiletés.

La première infirmière était née. Bien loin d'être en mesure de s'appuyer sur un corpus de connaissances cohérent, c'est intuitivement qu'elle alimenta d'amour, d'espoir, d'attention et de tendresse les fibres profondes du malade. Elle sentait que ces apports rendaient plus facilement assimilables les éléments vitaux qu'elle lui procurait en se substituant à lui pour combler une perte partielle ou totale, temporaire ou permanente d'autonomie. L'infirmière représentait, en quelque sorte, le malade par procuration vis-à-vis de sa famille, sa communauté et son environnement. C'était l'alter ego en santé du malade. Bien avant la lettre, l'infirmière considérait le malade comme un être biopsycho-social. À cette époque, selon Dolan (1978), le rôle de l'infirmière comprenait aussi ceux de nutritionniste, pharmacien, travailleur social et physiothérapeute.

\section{L'infirmière et le médecin}

\section{$\underline{\text { Retour à la table des matières }}$}

Contrairement à l'infirmière, fondamentalement orientée vers le malade et ses besoins de vie, le médecin, pour sa part, jouait un rôle situé au confluent de la magie et de la religion. Ses habiletés, accompagnées de rituels, visaient à extirper les mauvais esprits, ancêtres de la maladie, du corps du malade. D'une part, l'infirmière préservait, maintenait ou restaurait l'énergie vitale permettant l'harmonie de l'homme dans sa relation avec lui-même et avec son environnement et, d'autre part, le vide laissé par l'extraction de la maladie par le médecin permettait à l'énergie ainsi libérée de circuler de façon à participer à l'équilibre total du malade. Si Dolan considère qu'à cette époque la 
médecine était un prolongement du rôle joué par l'infirmière, c'est sans doute à ce moment que leur relation de complémentarité a vu le jour.

La perception du rôle de l'infirmière a varié à travers les âges en fonction de la valeur que les grandes civilisations anciennes octroyaient à la vie, à la santé et au statut de la femme. Plus souvent qu'autrement, l'infirmière, au statut social très bas, quand elle n'était pas esclave, n'avait pas le choix d'accepter un rôle qui s'apparentait davantage à celui de servante qu'à celui d'infirmière. Il n'y a guère que dans l'ancienne culture hébraïque que l'infirmière jouissait d'indépendance. Dolan souligne que, dans cette culture, l'infirmière jouait un rôle de prévention et de maintien de la santé physique et mentale et ce, tant dans son approche familiale que communautaire.

Si la mythologie grecque a permis à l'infirmière, sous les traits de la déesse Hygie, de prendre sa place dans l'équipe de base en santé, Hippocrate, pour sa part, fut le premier à plaider en faveur de la préparation académique adéquate de l'infirmière.

\section{L'influence du christianisme}

$\underline{\text { Retour à la table des matières }}$

Rompant avec la tradition gréco-latine, l'avènement du christianisme mit en relief la dignité fondamentale de tous les hommes. Cette philosophie eut pour conséquence, entre autres, de promouvoir, dans une certaine mesure, l'émancipation de la femme et l'élévation de son statut.

Plusieurs femmes de l'élite se convertirent au christianisme. Le soin des malades étant un moyen de marcher dans les traces du Christ pour atteindre le salut éternel, ces dernières, en plus de prodiguer des soins imprégnés de charité, apportèrent à l'organisation des soins tous les avantages de leur haute éducation. C'est ainsi qu'elles devinrent, selon Dolan, des réformatrices sociales. Elles étendirent leur action 
dans tous les domaines où les besoins de santé existaient. Ces infirmières fondèrent et administrèrent des hôpitaux, prodiguèrent des soins de façon indépendante bien qu'en étroite collaboration avec les autres membres de l'équipe de santé quand cela était nécessaire. De plus, elles surent partager connaissances et habiletés avec des néophytes animés d'un même esprit charitable.

Puis, vint la fondation de plusieurs ordres monastiques qui, en plus d'offrir à leurs adhérents la vie religieuse qu'ils recherchaient, plaçaient très haut dans leurs priorités le soin aux malades. Plus tard, des femmes repenties et des veuves formèrent des groupes charitables se dévouant, conjointement avec les religieuses, pour répondre, dans un même esprit spirituel et humanitaire, aux besoins de la société en temps de persécutions, de guerres et d'épidémies. La découverte des Amériques allait offrir un nouveau champ d'action à ces femmes.

À la Renaissance, en France, saint Vincent de Paul et sa nouvelle conception de la charité : "Aider les gens à s'aider eux-mêmes ", donnèrent à la dimension sociale une place stratégique dans le soin global des malades. Fondateur de plusieurs organisations charitables, saint Vincent de Paul était cependant convaincu qu'une charité mal orientée pouvait faire plus de tort que de bien. Rapidement, il ressentit que les soins qu'il préconisait devaient être enseignés, dirigés et coordonnés. Il confia donc à sainte Louise de Marillac la création du premier programme d'éducation systématique en soins infirmiers.

Les infirmières participèrent à l'aventure des pionniers du nouveau monde. C'est dans des conditions des plus précaires que les Soeurs Augustines et Jeanne Mance fondèrent les premiers hôpitaux de la Nouvelle-France. Indispensables et toujours animées d'un même élan charitable, elles furent héroïques dans leurs combats, tant en temps de guerre qu'en temps d'épidémie.

Le XVIlle et le début du XIXe siècle furent marqués par les guerres, les révolutions et les épidémies. Si les sciences et la médecine continuèrent à jeter leurs assises en continuité avec la Renaissance, il n'en fut pas de même pour les soins infirmiers. En effet, selon Nutting et Dock (1935), les soins infirmiers et l'organisation des hôpitaux se détériorèrent grandement au XVIlle siècle. Au début du XIXe siècle, 
les hôpitaux, hormis ceux dirigés par les religieuses, avaient très mauvaise presse. Étant presque exclusivement réservés aux nécessiteux, les soins y étaient souvent donnés par des hospitalisés de cinquante, voire même de quatre-vingts ans. Faut-il s'étonner d'entendre parler de soins négligés, de mauvais traitements des malades et du taux élevé de mortalité dû à l'infection dans les hôpitaux de l'époque ? Devant cet état de choses, ce sont les mères qui ont à nouveau assumé la responsabilité des soins.

\section{De la lampe au phare : Florence Nightingale}

La lumière apportée par Florence Nightingale ne fut un luxe ni pour le malade ni pour les soins infirmiers. Cette Anglaise hautement intelligente et éduquée s'est illustrée, entre autres, par sa détermination à vaincre les oppositions de toutes sortes dans le but d'améliorer la qualité et l'organisation des soins lors de la guerre de Crimée. Sa perspicacité et sa capacité de pénétrer et d'évaluer la situation des soins lui permirent d'analyser les problèmes de façon à leur opposer des solutions efficaces tant pour l'amélioration des conditions sanitaires que pour la qualité des soins et ce, aussi bien en temps de guerre qu'en temps de paix. Sa philosophie, avant-gardiste à l'époque, mais encore bien d'actualité, consistait à considérer que c'était l'individu dans sa totalité que l'infirmière devait soigner et non la maladie. Les soins infirmiers étaient, pour elle, l'ensemble des soins mettant l'individu dans les meilleures conditions possibles pour que la nature restaure ou préserve la santé, prévienne ou guérisse les maladies ou les blessures. Les deux grands axes des soins infirmiers se dégagent clairement de cette définition : d'une part, les soins aux individus en santé qui, selon Florence Nightingale, consistaient à garder ou mettre une personne saine dans des conditions pour éviter la maladie et, d'autre part, les soins aux malades qui consistaient à les aider à vivre une vie aussi pleine et satisfaisante que possible. Il va sans dire que cette femme croyait fer- 
mement que c'est sur l'infirmière que repose la responsabilité de la santé, du confort et du bien-être tant de l'individu que de la société.

Jamais Florence Nightingale n'a accepté que des pertes de vie puissent être causées par la négligence et l'ignorance. Dès ses débuts, selon Dolan, elle insistait pour que des femmes des plus intelligentes et compétentes prodiguent les soins. Elle n'aurait jamais toléré dans son équipe de soins des femmes sans formation adéquate, aussi dévouées et soumises qu'elles pussent avoir été. Forte de son expérience dans la fondation d'écoles pour soldats, ambulanciers, cuisiniers et de son rôle d'instigatrice dans la fondation d'une école de médecine pour officiers, elle voulut fonder sa propre école d'infirmières, tout imprégnée de sa philosophie des soins, qui avait déjà fait ses preuves.

Les grands buts de son école seraient, bien entendu, autant d'apprendre aux étudiantes à donner des soins préventifs qu'à administrer des soins aux malades, tant à l'hôpital que dans la communauté. De plus, cette école préparerait les infirmières pour l'enseignement des soins infirmiers. Elle-même d'une haute éducation, elle insistait sur l'importance d'une éducation avancée pour assumer les responsabilités d'infirmière. Florence Nightingale était d'opinion que les soins infirmiers impliquent un corpus de connaissances et un rôle différents de ceux de la médecine. Pour elle, selon Dolan, infirmière et médecin étaient des collègues travaillant ensemble mais ayant des fonctions différentes.

Le projet éducatif de Florence Nightingale et surtout sa philosophie de base ne furent pas sans créer une vive opposition de la part de la centaine de médecins consultés avant la fondation de l'école. Dolan rapporte l'opinion de certains d'entre eux : « Les infirmières jouent un rôle de femmes de chambres ; elles n'ont guère besoin de plus de formation que pour fabriquer des cataplasmes, maintenir la propreté et répondre aux désirs des malades ». "Une infirmière est une servante digne de confiance, mais une servante tout de même... Elle devrait être d'âge moyen pour commencer à exercer en soins infirmiers, et si elle a été asservie par le mariage et les problèmes familiaux, ce n'en sera que mieux. » Le mépris n'impressionnant pas Florence Nightingale, elle déclara : "Une infirmière ne devrait rien faire d'autre que soigner. Si vous voulez des femmes de ménage, engagez-en. Les soins 
infirmiers sont une spécialité ». Florence Nightingale ouvrit son école d'infirmières !

Le sens aigu de l'observation de cette femme l'avait convaincue que le cadre organisationnel dans lequel évoluaient les infirmières affectait le soin aux malades. Il fallait organiser les soins de façon à permettre aux infirmières de se consacrer exclusivement aux soins. Dans ce but, elle proposa que les tâches subsidiaires soient réservées à des travailleurs moins scolarisés. En plus d'atteindre son objectif, cette proposition s'avérait moins coûteuse que le statu quo. Florence Nightingale fut une administratrice et une réformatrice d'hôpitaux inégalée à son époque. Selon Dolan, son livre Notes on Hospitals fut considéré comme l'oeuvre la plus pratique, la plus scientifique sur la construction, l'organisation et l'administration des hôpitaux.

Même si aujourd'hui plusieurs sont étonnés d'apprendre que des infirmières font de la recherche, ils le sont encore davantage lorsqu'ils apprennent qu'en cela, ces infirmières marchent dans les traces de Florence Nightingale qui était elle-même un chercheur chevronné. Ses réformes dans tous les domaines ne furent pas le fruit de caprices mais bien le résultat d'un esprit indépendant et créateur qui savait s'appuyer sur de méticuleuses investigations et de solides statistiques.

Dolan est convaincue que sans les réformes de Florence Nightingale dans les domaines des soins infirmiers et des hôpitaux, le triomphe de la médecine et de la chirurgie modernes auraient été impossibles. C'est peut-être ce qui a fait dire à un médecin anglais du nom de Saleeby : « Si Lister a été le père de la chirurgie, Florence Nightingale en fut la mère ».

Femme supérieure, ardente avocate des droits des femmes, par sa philosophie et son oeuvre, Florence Nightingale a convaincu des milliers de femmes que leur rôle d'infirmière était essentiel pour la société. Cette femme du XIXe siècle savait-elle qu'elle posait, pour les infirmières, les deux premiers jalons qui, selon Bixler et Bixler dans Dolan, constituent l'essence même d'une profession : utiliser, dans la pratique, un corpus bien défini et bien organisé de connaissances spécialisées provenant d'un niveau intellectuel supérieur ; augmenter sans 
cesse son corpus de connaissances, améliorer ses techniques éducatives et sa pratique par l'utilisation de la méthode scientifique.

On pourrait croire qu'avec l'héritage laissé par Florence Nightingale, l'évolution à claire-voie des soins infirmiers et, par là, celle des infirmières, était terminée. Leurs hoquets, voire leurs soubresauts, continuèrent au contraire et continueront encore parce qu'ils s'insèrent dans le grand mouvement de la nature.

\section{Soigner : ancrer à la vie}

$\underline{\text { Retour à la table des matières }}$

Soigner, c'est d'abord et avant tout, selon Collière (1982), un acte de vie. C'est peut-être parce que les soins touchent l'essence de cette vie que la société, pour se rassurer, s'est créé des images mentales de l'infirmière, niant le caractère fondamentalement profond de sa profession. La belle infirmière (avez-vous déjà imagine une infirmière laide ?) qui sauve héroïquement des vies avec autant de succès qu'un pêcheur qui tend ses filets dans un banc de poissons - c'est l'infirmière active - ou encore la belle infirmière, humblement et corps et âme dévouée à la gloire du médecin qui la laisse béate d'admiration - c'est l'infirmière contemplative.

Personne n'entend le coeur de l'étudiante infirmière battre trop fort quand elle sent étouffer les élans d'héroïsme de son âge en entrant dans ces gigantesques machines distributrices de soins que sont les hôpitaux. Personne ne voit son vertige quand elle prend conscience que ses sens s'anesthésieront dans une chaleur tropicale aux odeurs stériles où le feutre des mots et des pas ne ranime en rien les couleurs mortes et les gestes à la fadeur d'un fruit trop froid. Si cette famine des sens constitue une clause cachée dans les conditions de travail de l'infirmière, pour le malade, elle correspond à l'image du prix qu'il croit devoir payer pour recevoir des soins. 


\section{Des soins avec ou sans bon sens}

$\underline{\text { Retour à la table des matières }}$

Dans les centres qui ont la santé pour objectif, on oublie trop souvent que la santé est normale et que quelle que soit la gravité de la maladie d'un individu, il reste toujours des aspects sains qui le relient à la vie. Les stimulations sensorielles maintiennent le contact avec l'environnement, avec la vie. Pour quelle raison les dévitaliser, les rendre superflues sinon anormales?

Réduire la chaleur languissante des centres de santé aurait un effet bénéfique sur la note de chauffage, revigorerait les malades et le personnel, sans compter qu'une diminution des changements brusques de température durant l'hiver pourrait probablement réduire le nombre de jours de maladie du personnel.

En ce qui a trait à l'odorat, pourquoi ne pas personnaliser les pièces des centres de santé au moyen d'un pot-pourri d'herbes aromatiques aux parfums subtils et délicats ; bien des diaphragmes s'y laisseraient couler de plaisir au lieu d'offrir leurs rigides résistances aux effluves chimiques.

S'il est vrai que les malades ont besoin de calme, ils n'ont cependant pas besoin de la constance du vide qu'ils ne connaissent pas dans leur vie quotidienne. Pourquoi ne pas diffuser des enregistrements de chants d'oiseaux, de vagues d'océan, de pluie qui tombe doucement et même de rires d'enfants ? Combien cela serait relaxant pour tout le monde et davantage près de la vie.

L'art naît de la vie et y retourne. Pourquoi ne pas l'accrocher aux murs des longs corridors ? L'idée n'est pas nouvelle ; au Moyen Âge, selon Dolan, c'était la coutume de disposer des peintures aux murs des hôpitaux dans le but de distraire les malades. Non seulement des oeuvres, qu'elles soient des malades, du personnel ou d'artistes, pourraient distraire, égayer et permettre le développement du sens esthétique 
mais, ce qui n'est pas à négliger, par le biais des oeuvres exposées au public, la population se sensibiliserait au grand esseulement de plusieurs de ses malades.

Le domaine du goût est celui que le malade se permet le plus de sentir lésé. Un plat savoureux donne l'impression de vivre. S'il est impossible de recréer les saveurs familières affectionnées par chaque malade, un soupçon d'imagination allié à des aromates bien dosés pourraient transformer le repas, de moment difficile mais nécessaire, en moment de plaisir attendu.

S'il est un type de repas absolument exempt de sensualité gustative, c'est bien les médicaments. Ce n'est un secret pour personne, notre société est surmédicamentée. Combien de malaises légers se résorbent d'eux-mêmes et combien proviennent de relations malsaines entre l'individu et son corps, l'individu et son environnement. Les gens ont oublié le langage du corps et son décodage. Quand une céphalée se présente, ils n'entendent pas leur corps leur dire : «J'en ai assez d'étouffer dans cet air vicié, donne-moi de l'air pur et frais » ou encore « tous ces problèmes me tendent les nerfs comme des cordes de violon, je suis tellement concentré sur eux que j'en oublie presque de respirer » - « s.v.p. respire donc profondément, bouge tous tes membres pour refaire circuler l'énergie bloquée ».

On a conditionné l'homme de telle sorte qu'il considère qu'un malaise doit enclencher automatiquement sa dépendance vis-à-vis de la chimie magique. Le contrôle de son corps ne lui appartient plus, il appartient à des formules hiéroglyphitiques qui, après quelques tours de passe-passe, atterrissent dans son organisme d'où, en bon spectateur, l'individu jouit du miracle attendu. Ce qui est inquiétant dans cette situation, c'est que tout vrai magicien ne croit pas à la magie. Il sait qu'il crée une illusion et que la magie est dans l'oeil du spectateur. Dans le cas qui nous occupe, les magiciens de la chimie croient cependant fermement à leur magie... Cette foi dans la chimie des poisons comporterait-elle plus d'avantages que l'interprétation et l'enseignement au malade du langage de son corps ? Loin de moi l'idée de nier l'importance des médicaments ; dans bien des cas, des vies en dépendent. Ce qu'il faut cependant éviter de croire et de faire croire, c'est que l'actuel réflexe de leur absorption appartient à la nature de 
l'homme. Il est tout à fait inacceptable que, pour certains individus, la seule occasion de boire un verre d'eau soit pour les aider à avaler leurs médicaments. Les centres de santé, où les médications du sommeil sont presque automatiques, devraient eux-mêmes donner l'exemple. Pourquoi ne pas d'abord évaluer le sommeil des malades avant de leur prescrire des hypnotiques. Devant un réel problème de sommeil, pourquoi ne pas donner avant le coucher une infusion bien chaude d'aneth, d'angélique, de camomille ou de tilleul qu'un médecin aura pris soin de recommander et de prescrire ? Ces infusions, par leur chaleur et leur saveur, pourraient constituer des éléments agréables dans un rituel favorisant le sommeil. De plus, ces infusions pourraient être le début du désamorçage d'une dépendance autorisée aux médicaments.

Tranquillement, on redécouvre que le toucher est un moyen de communiquer. Il y a bien ces gestes rapides et fonctionnels qui sont trop souvent confondus, par la population, avec l'essence de la profession infirmière. Mais il y a surtout la chaleur d'une main calme qui irradie sa présence jusqu'au coeur ; il y a ces deux mains posées sur les épaules qui savent si bien camper la confiance, et que dire de cette main offerte à la main crispée pour en partager l'angoisse douloureuse ? Les malades sont dépouillés de leurs gestes quotidiens dans les centres de santé. Pourquoi ne pas offrir une petite plante, qu'il ferait croître, au malade hospitalisé en psychiatrie ou pour des soins à long terme ? De plus, pour quelle raison n'aurait-il pas la responsabilité de petits animaux ?

Si l'on considère que la population a droit à l'information, nous sommes forcés de nous poser des questions sur le statut des malades par rapport à cette dernière quand nous constatons l'absence navrante de journaux ou revues accessibles gratuitement pour ces derniers.

Ces quelques pistes de réflexions et réfections ne veulent pas transformer les centres de santé en palaces mais bien rattacher la vie précieuse en chaque malade à la source vivifiante de son quotidien afin qu'il et qu'on ne considère plus la maladie comme une parenthèse l'extrayant du courant de la vie, comme un maillon béant sans contact aucun avec le passé et l'avenir qui le font homme. Faut-il obligatoirement avoir recours à une unité de soins palliatifs pour que soient res- 
pectés les sens qui participent à la qualité de la vie de façon à ne pas, comme le dit si bien Collière, «mourir sa vie ».

La gestion des centres de santé devrait être aménagée de façon à permettre à l'infirmière de prodiguer en priorité les soins fondamentaux reliés à l'entretien et au maintien de la vie des malades. Selon Collière, ces soins

représentent tout cet ensemble d'activités qui assurent la continuité de la vie comme : boire, manger, évacuer, se laver, se lever, bouger, se déplacer, ainsi que tout ce qui contribue au développement de la vie de notre être, en construisant, en entretenant l'image du corps, le réseau relationnel, en stimulant les échanges avec tout ce qui est fondamental à la vie, car source d'énergie vitale : la lumière, la chaleur, la relation avec les personnes connues, les objets familiers, etc.

Ces soins nécessitent, de la part de l'infirmière, une souplesse tenant compte des besoins, de la culture et des habitudes de vie du malade de façon à maintenir le plus possible l'intégrité de la trame de vie de ce dernier. Par cette souplesse, il y a reconnaissance de l'unicité de chaque malade mais elle ne peut s'inscrire que dans un cadre partageant la même philosophie.

\section{Une expérience à tenter}

$\underline{\text { Retour à la table des matières }}$

Ceci m'amène à vous faire faire une expérience. À partir de demain, quatorze heures, et pour un temps indéterminé, changez complètement vos heures de repas, votre menu habituel, changez vos heures pour faire votre toilette de même que vos heures de coucher et de lever; bien sûr, changez aussi vos vêtements et ne portez que de grands sacs de coton blancs complètement ouverts à l'arrière. Imaginez (uniquement imaginer...) qu'à partir de ce moment, vos paroles n'ont d'échos que pour vous. Elles se transforment en pistes solides ou liquides stimulant la vigilance de prospecteurs qui vous sont inconnus. Vous devenez une mine souterraine où un bataillon de mineurs fouillent vos veines et vos entrailles pour en extraire des éléments dont la valeur n'a de sens que dans un langage qui vous est étranger. 
Si vous avez assez de courage, imaginez-vous à votre réveil d'un long et profond sommeil. Vous êtes l'hôte de multiples tentacules, excroissances de boîtes électriques abreuvant leurs voyants lumineux et leurs chants électroniques à la chimie et la physique de votre corps leur tenant lieu de laboratoire ... Cette expérience imaginaire n'est pas facile. Pour bien des malades, cependant, elle n'a rien d'imaginaire. Pour être ainsi propulsé dans cette enclave de la maladie qui agresse si sournoisement l'harmonie qui leur est propre, les malades doivent présenter de solides prérequis d'une capacité d'adaptation à toute épreuve. Et pourtant, les scientifiques ont jugé indispensable de donner un entraînement intensif aux cosmonautes pour leur apprendre à vivre et survivre dans un environnement aux conditions étrangères à leur vie quotidienne...

Rassurez-vous, l'expérience est bien terminée et pour être malade, vous n'avez pas (encore) besoin de diplôme d'une école d'adaptation aux centres de santé. Toutefois, le traitement de la maladie requiert des soins s'attaquant directement aux digues qui entravent la libre circulation de la vie. Pour Collière, ces soins sont les soins de réparation. Il ne faut cependant pas perdre de vue que ces soins n'ont de sens qu'à la condition que les soins fondamentaux, d'entretien et de maintien de la vie, mobilisent chez le malade suffisamment d'énergie vitale pour qu'il se dispose à les recevoir. Selon Collière, la vie se retire à chaque fois que l'on se préoccupe davantage de ce qui meurt que de ce qui vit.

\section{L'infirmière en devenir}

$\underline{\text { Retour à la table des matières }}$

Depuis Florence Nightingale, le rôle de l'infirmière s'élargit sans cesse. Il ne faut pas croire cependant que cette ampleur croissante doive engendrer pour autant la dilution de son essence. La santé, par le biais de la mobilisation de l'énergie vitale de l'individu est, et doit rester, le coeur des préoccupations professionnelles de l'infirmière. Cependant, la population et les services de santé restent prisonniers d'images qui tendent à édulcorer, chacune à sa manière, l'objectif premier de la profession infirmière. 
En faisant le choix de devenir infirmière, l'étudiante ignore que sa douce image d'ange blanc thaumaturge volera bientôt en éclats. Souvent elle est étonnée sinon déçue de constater que les habiletés techniques cèdent le pas devant les habiletés à la relation d'aide constituées principalement d'écoute et d'empathie plutôt que d'actions et de sympathie. L'image de l'infirmière compétente ne tourne-t-elle pas autour d'une seringue et d'une aiguille ? Elle ignore au départ qu'elle devra devenir polyglotte afin d'être une traductrice-interprète efficace entre le langage verbal et non verbal de chaque malade et de sa famille et les différents membres de l'équipe de santé. C'est elle qui, par la suite, traduit le langage scientifique (qui possède l'art de dramatiser le moindre rhume de cerveau) en un langage ayant une signification à la portée de chaque malade et de sa famille. Elle doit aussi apprendre le langage du corps propre à chaque malade et à chacun apprendre à décoder ce langage oublié, expression de bien des vérités profondes.

Il n'est pas facile pour l'étudiante d'accepter que l'image de l'activisme instrumental, de la bonne infirmière qui fait tout pour le malade, doive se transformer pour faire place à l'écoute, la réflexion, l'identification des besoins, la planification et l'évaluation des soins et ce, tant dans une optique préventive que curative. Comme, dans le domaine de la santé, l'image populaire ne réserve qu'aux seuls médecins les longues études scientifiques, l'étudiante comprend difficilement, au début, qu'en plus d'être différent de ce qu'elle croyait, l'étendue et la complexité de son rôle, tributaire de l'immense complexité de l'homme, exigent qu'elle greffe solidement sa profession sur I'intégration de connaissances approfondies tant en sciences pures qu'en sciences humaines. Rapidement, elle comprendra que nier cette exigence équivaut à prendre un billet « aller seulement » pour le monde de l'incompétence et le statut de servante ignare dans lequel les opposants de Florence Nightingale voulaient maintenir les infirmières il y a un siècle.

Ces combats pour maintenir intactes les images qu'elle possède sont nécessaires à l'étudiante et font partie, selon Davis (1972), de la socialisation professionnelle des infirmières. Pour devenir infirmière, et non la concrétisation d'une image, l'étudiante doit hélas ! perdre tous ses combats tout en restant elle-même. Ce n'est qu'avec un certain 
recul qu'elle pourra conclure, malgré tous ses deuils, qu'elle a joué à « qui perd gagne ».

\section{Non à la profession omnibus}

\section{$\underline{\text { Retour à la table des matières }}$}

Le repos de l'infirmière n'aura pas lieu. Si elle a combattu, pendant sa formation, les images que la société voulait lui imposer, dès son entrée dans le circuit des services de santé, elle est trop souvent confrontée à l'évidence que la guerre des images n'est pas terminée. C'est effectivement très tôt qu'elle se rend compte, par le type de demandes qui lui sont faites, qu'il y a erreur sur la personne. Elle qui se veut infirmière, on veut lui faire exercer une sorte de profession omnibus pour laquelle ni elle, ni personne n'est préparé. Qui, en effet, possède la préparation pour, à la fois diriger la circulation place de la Concorde à l'heure de pointe, éteindre en même temps tous les incendies, remplacer temporairement sinon à plein temps la secrétaireréceptionniste, gérer dans certaines circonstances l'argent des malades et, bien sûr, faire tout ce qui précède en ménageant les susceptibilités de tout le monde. J'oubliais, elle doit aussi soigner les malades... Kyes, dans une récente conférence nationale sur la formation infirmière, mentionnait que l'écart entre la préparation professionnelle de l'infirmière et les attentes du milieu à son égard provoque chez elle une désillusion fort décevante en plus d'un désagréable sentiment d'être écrasée par le milieu.

Heureusement, un nombre croissant d'infirmières, dont l'évolution est, selon Dumont-Johnson (1981), intimement liée à celle de la femme, refusent de considérer et d'accepter qu'on considère leur profession comme un métier hybride exercé par des travailleuses non spécialisées. Elles refusent avec d'autant plus de conviction quand elles identifient clairement que la difficulté qu'on éprouve a percevoir qu'elles ont un rôle complet et précis à jouer auprès des malades découle de l'image éculée mais combien rentable du rôle traditionnel de la femme. En effet, c'était la femme qui, par une traditionnelle néces- 
sité, effectuait l'ensemble des tâches non spécialisées requises pour la bonne marche du foyer. L'allègre empressement des services de santé pour maintenir l'identification de l'infirmière à l'image sécurisante et économique de reine de foyer sans pouvoir, rencontre aujourd'hui un imposant barrage de femmes qui savent endiguer ces élans pour les ramener dans une perspective plus réaliste et plus respectueuse de la profession infirmière.

Tout comme la femme, l'infirmière apprend maintenant à refuser d'intérioriser des images qui ne correspondent en rien, aujourd'hui, à ce qu'elle est et au rôle pour lequel elle a été formée. Elle perçoit de plus en plus clairement la valeur réelle de sa profession dans le réseau économique. En nombre sans cesse croissant, les infirmières s'approprient des connaissances supérieures qui leur permettent de découvrir, tant pour elles que pour leurs malades, un éventail de pouvoirs qui leur semblaient jadis interdits parce qu'inaccessibles. De plus, la recherche en soins infirmiers favorise une attitude de réflexion indispensable pour donner au corpus de connaissances de la profession toute la cohérence nécessaire à une haute qualité de soins.

\section{Oui à la vie}

$\underline{\text { Retour à la table des matières }}$

Nés d'un élan naturel pour maintenir la vie, les soins infirmiers seront bientôt requis par une civilisation interplanétaire. Malgré leur complexité sans cesse croissante, leur objectif ultime sera toujours de maintenir et faire croître dans la dignité la moindre étincelle d'énergie vitale permettant à l'homme de s'arrimer à la vie. 


\section{Références}

$\underline{\text { Retour à la table des matières }}$

J.A. DOLAN, Nursing in Society, A Historical Perspective, Philadelphia, W. B. Saunders, 1978.

M.A. NUTTING and L. DOCK, A History of Nursing, New York, B.P. Putnam's Sons, 1935.

F. DAVIS, Illness, Interaction and the Self, Belmont, Cal., Wadworth, 1972.

J.J. KYES, The Clinical Component! Implications for Socialization into the Professional Role, conférence inédite, IIe congrès national de la formation infirmière, A.I.I.C., Winnipeg, 1982.

M.F. COLLIÈRE, Promouvoir la vie. De la pratique des femmes soignantes aux soins infirmiers, Paris, Inter Editions, 1982.

M. DUMONT-JOHNSON, " Les inrfirmières cols roses ? ", Nursing Quebec, 1, 6, 1981, pp. 10-16.

Fin du texte 